\title{
The promoter-proximal rDNA terminator augments initiation by preventing disruption of the stable transcription complex caused by polymerase read-in
}

\author{
Sheryl L. Henderson, Kenneth Ryan, and Barbara Sollner-Webb' \\ Johns Hopkins University School of Medicine, Department of Biological Chemistry, Baltimore, Maryland 21205 USA
}

\begin{abstract}
We have examined the mechanism by which transcriptional initiation at the mouse rDNA promoter is augmented by the RNA polymerase I terminator element that resides just upstream of it. Using templates in which terminator elements are instead positioned at the opposite side of the plasmid rather than proximal to the promoter, or conditions where transcription is terminated elsewhere in the plasmid by UV-induced lesions, we show that the terminator's stimulatory effect is not position dependent. Mouse terminator elements therefore do not stimulate via the previously postulated 'read-through enhancement' model in which terminated polymerases are handed off to an adjacent promoter in a concerted reaction. The position independence and orientation dependence of the terminator also makes it unlikely that the terminator functions as a promoter element or as an enhancer. Instead, terminators serve to augment initiation by preventing polymerases from reading completely around the plasmid and through the promoter from upstream, an event which we show interferes with subsequent rounds of initiation. Notably, this transcriptional interference arises because polymerase passage across a promoter disrupts the otherwise stable transcription complex, specifically releasing the bound transcription factor $D$. These liberated $D$ molecules can then bind to other templates and activate their expression. The rDNA transcriptional interference is not due to a steric impediment to the binding of new polymerase molecules, and it does not similarly liberate the initiationcompetent polymerase (factor $\mathrm{C}$ ). These studies have also convincingly demonstrated that multiple rounds of transcription are obtained from rDNA template molecules in vitro.
\end{abstract}

[Key Words: Transcriptional interference; promoter occlusion; termination; rDNA transcription; RNA polymerase I; stable complex]

Received August 19, 1988; revised version accepted December 12, 1988.

Ribosomal RNA genes reside in large tandem arrays in the genomes of all higher eukaryotes. We and others have identified RNA polymerase I 'terminator' elements just upstream from the rRNA gene promoter in a number of metazoan species (Morgan et al. 1983; Moss 1983; Grummt et al. 1986; Henderson and Sollner-Webb 1986). These terminator elements have the curious property of augmenting transcription from an adjacent polymerase I promoter (Grummt et al. 1986; Henderson and Sollner-Webb, 1986; McStay and Reeder 1986; DeWinter and Moss 1987). The mechanism(s) by which these elements stimulate transcription remains unclear. It has frequently been suggested that a terminator may function in 'read-through enhancement' (Moss 1983; Henderson and Sollner-Webb 1986; McStay and Reeder 1986; Mitchelson and Moss 1987), a process envisioned to involve termination and concerted reinitiation at the adjacent promoter by polymerases that read into that region from upstream. Alternatively, the presence of the

${ }^{1}$ Corresponding author. terminator may favor initiation by preventing promoter occlusion or transcriptional interference, i.e., by disengaging polymerases that would otherwise inhibit initiation due to their passage through the promoter /Adhya and Gottesman 1982; Cullen et al. 1984; Proudfoot 1986). Such promoter occlusion is generally envisioned to occur because the traversing polymerases block productive attachment of new initiating polymerases, but it could also occur because the traversing polymerases destabilize the binding of the transcription initiation factor(s) to the promoter. Certainly, terminators could also augment initiation by a combination of these or other means. In this paper, we show that the major mechanism of transcriptional stimulation by the rodent promoter-proximal terminator is the prevention of transcriptional interference. Specifically, we demonstrate that polymerase molecules that read through the stable transcription complex on the mouse rDNA promoter destabilize that association. The promoter-binding rDNA transcription factor D is released and is able to transfer to another template molecule where it can direct additional rounds of transcription, while the en- 
gaged active polymerase (factor C) does not similarly transfer. Controls demonstrate that the transfer of factor $\mathrm{D}$ indeed requires transcription through the promoter complex and that it is not the initiation event or transcription per se that causes this effect. We therefore conclude that the major role of the promoter-proximal terminator of the mouse rRNA gene is not to facilitate sequestration of polymerases as had been previously proposed, but rather to protect the active promoters from the dissociation of transcription factor $\mathrm{D}$. While this paper was under review, experiments showing an analogous disruption of the Acanthamoeba rDNA initiation complex by polymerase read-in were published $(\mathrm{Ba}$ teman and Paule 1988).

\section{Results}

Transcriptional stimulation by the terminator element is position independent

We earlier demonstrated that the sequence immediately downstream of residue -170 of the mouse rRNA gene serves to terminate RNA polymerase I transcripts that read into this region from upstream (Henderson and Sollner-Webb 1986). Unexpectedly, this terminator element was also found to augment the level of transcriptional initiation at the adjoining rDNA promoter relative to that from a similar construct which lacks this element. Data illustrating this result are reproduced in Figure 1. Closed circular plasmid templates carrying rDNA segments beginning 230,168, or 163 residues upstream of the initiation site were transcribed in transiently transfected cells (part A) or in an S-100 cell extract (part B). The resultant RNAs were analyzed by S1 mapping using a probe that is $5^{\prime}$-end labeled within the rRNA coding region and diverges in sequence from the

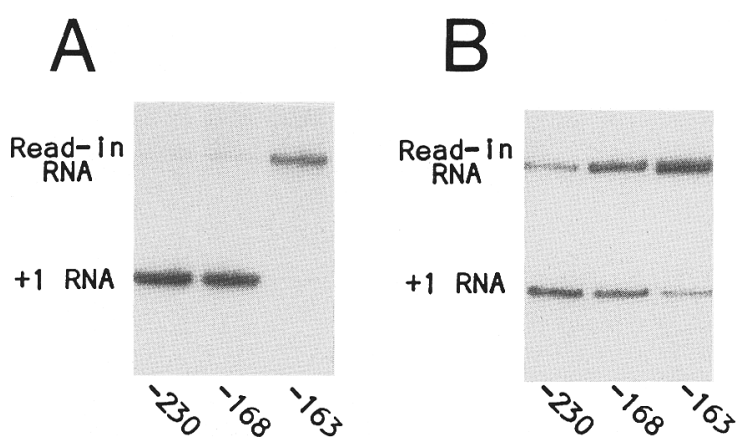

Figure 1. Transcription of $5^{\prime}$ deletions of the mouse rDNA promoter in vivo and in vitro. The indicated $5^{\prime}$ deletion templates were allowed to transcribe in transiently transfected rodent cells (panel $A$ ) or in an in vitro reaction with S-100 extract (panel $B$ ), and the resultant RNA was detected by S1 analysis, using a probe that is $5^{\prime}$ end labeled at residue +292 and diverges in sequence from the template at residue -168 or the site of the deletion (diagramed in Henderson and Sollner-Webb, 1986; Fig. 1A). Transfections were performed at a DNA concentration of $2 \mu \mathrm{g} / \mathrm{ml} .(+1$ RNA) RNA that initiated at the natural rRNA start site; (Read-in RNA) all RNA that reads into the promoter region from upstream.
rDNA at residue - 168 . All RNA that reads into the initiation region from upstream thus protects the probe up to the site of the divergence, yielding a single 'read-in

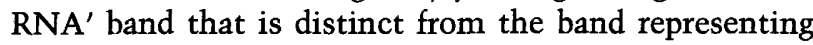
correct transcriptional initiation at residue +1 (Fig. 1). This read-in RNA results almost entirely from polymerases that initiated at the rDNA promoter and read completely around the plasmid, because it is virtually abolished by mutations that impinge on the core promoter domain (Henderson and Sollner-Webb 1986).

In transiently transfected cells (Fig. 1A), plasmids $5^{\prime} \Delta-230$ and $5^{\prime} \Delta-168$ (which contain functional terminator elements) direct efficient initiation with negligible amounts of read-in RNA (lanes 1 and 2), while $5^{\prime} \Delta-163$ and smaller templates / which lack important terminator sequences but contain the core promoter) direct much lower levels of correct initiation; this RNA extends around the entire plasmid more than one time to yield a strong read-in signal (lane 3). The same qualitative effects of the -168 region are also observed in vitro (Fig. $1 \mathrm{~B})$. In vivo, the -168 region has a very large transcriptional stimulatory effect and is very efficient at terminating transcripts that approach the initiation region from upstream (part A), while in vitro (part B) this termination efficiency ranges from $70 \%$ downward (Henderson and Sollner-Webb 1986), depending on the conditions of the reaction and the batch of cell extract.

To account for the apparent transcriptional stimulation by the -168 terminator region, several classes of models should be considered. The first is a 'polymerase hand-off' or 'read-through enhancement' model in which polymerases that approach the promoter region from upstream are acted on by the promoter-proximal terminator element and are thereby positioned to favor a concerted reinitiation at that promoter (Moss 1983; Henderson and Sollner-Webb 1986; McStay and Reeder 1986; Mitchelson and Moss 1987). The second class of proposals, the 'brick wall model,' envisions that the -168 sequence is actually a promoter element to which a trans-acting factor binds and that this bound transcription factor also blocks progression of polymerase, thus causing the apparent termination (Baker and Platt 1986). The final class of models, which can be termed 'transcriptional interference' or 'promoter occlusion,' hypothesizes that initiation at a promoter is inhibited when polymerases transcribe through that promoter from upstream, and that an intervening terminator stops such incoming polymerases, thereby increasing the level of transcription relative to that obtained in the absence of the terminator. While it is generally envisioned that polymerases traversing a promoter can occlude further initiations by sterically blocking the attachment of new polymerase molecules (Adhya and Gottesman 1982; Cullen et al. 1984; Proudfoot 1986), an alternative possibility is that their passage would disnupt the stable association of promoter-binding transcription factors.

The first two classes of models predict that the terminator element would need to be located close to its natural promoter-proximal position in order to function either in handing off the polymerase to the promoter (model 1) or as a promoter element (model 2). In con- 
trast, the third class of models predicts that the terminator would exert its transcriptional stimulatory effect when positioned virtually anywhere within the plasmid, since in order to occlude initiation, polymerases would have had to traverse the entire molecule.

To exploit this distinction experimentally, polymerase I terminator elements have been moved to alternate locations in the rDNA plasmid. For the experiment of Figure 2A, a segment containing the natural rRNA 3' terminator region was inserted 155 base pairs beyond the initiation site of the $5^{\prime} \Delta-163$ template, which lacks the promoter-proximal terminator element (see Methods). Upon transfection into rodent cells and S1 analysis of the resultant RNAs, this plasmid supports a far greater level of initiation than the parental $5^{\prime} \Delta-163$ (compare the +1 RNA in lane 3 versus lane 2). (As discussed below, this plasmid actually directed slightly more transcription than did $5^{\prime} \Delta-230$, which contains an intact promoter-proximal terminator element (lane 1).] These data indicate that the polymerase I terminator's ability to stimulate transcription is not dependent on being located adjacent to the promoter.

To verify the position independence of the terminator's stimulatory effect, we constructed additional templates in which the $3^{\prime}$ terminator element was positioned further around the plasmid. In these constructs the resultant transcripts would terminate $800 \mathrm{bp}$ downstream (or $3.7 \mathrm{~kb}$ upstream) of the initiation site. The resultant $5^{\prime} \Delta-163 / 3^{\prime} \mathrm{T}$ plasmid also directs much more initiation than does $5^{\prime} \Delta-163$ (Fig. 2B, lanes 5 versus 2). This confirms the conclusion of Figure $2 \mathrm{~A}$ that the tran- scriptional stimulation is not dependent on the position of the terminator element. A result qualitatively similar to that of Figure $2 B$ has also been observed in vitro when these templates were transcribed in cell extracts but, consistent with the lower efficiency of in vitro termination (Fig. 1B), the magnitude of the effect was smaller (data not shown).

The data of Figure 2B (and virtually identical results from other experiments) also lead to two additional conclusions. First, the observation that $5^{\prime} \Delta-163 / 3^{\prime} \mathrm{T}$ transcribes as efficiently as the analogous plasmids $5^{\prime} \Delta-230 /$ $3^{\prime} \mathrm{T}$ and $5^{\prime} \Delta-167 / 3^{\prime} \mathrm{T}$ (lanes 3 and 4 ) demonstrates that the presence or absence of a promoter-proximal terminator element has no influence on the level of initiation if polymerases have already been stopped prior to reading into that region from upstream. Second, the result that the $5^{\prime} \Delta-230$ template directs the accumulation of fewer transcripts than does $5^{\prime} \Delta-163 / 3^{\prime} \mathrm{T}$ and $5^{\prime} \Delta-230 / 3^{\prime} \mathrm{T}$ (Fig. $2 B$, lane 1 versus lanes $3-5$; see Discussion) indicates that the initiation rate is not increased if polymerases transcribe up to the promoter-proximal terminator element (as in $5^{\prime} \Delta-230$ ) rather than terminate $3.7 \mathrm{~kb}$ upstream from this site (as in the 3'T constructs). Together the data of Figures $2 \mathrm{~A}$ and $\mathrm{B}$ argue strongly against polymerase hand-off (read-through enhancement) having any significant effect on the level of transcription in our mouse rDNA expression system. They further demonstrate that the -168 region does not contain an important promoter domain whose function requires linear proximity to the initiation site. Instead, these data support a transcriptional interference model.

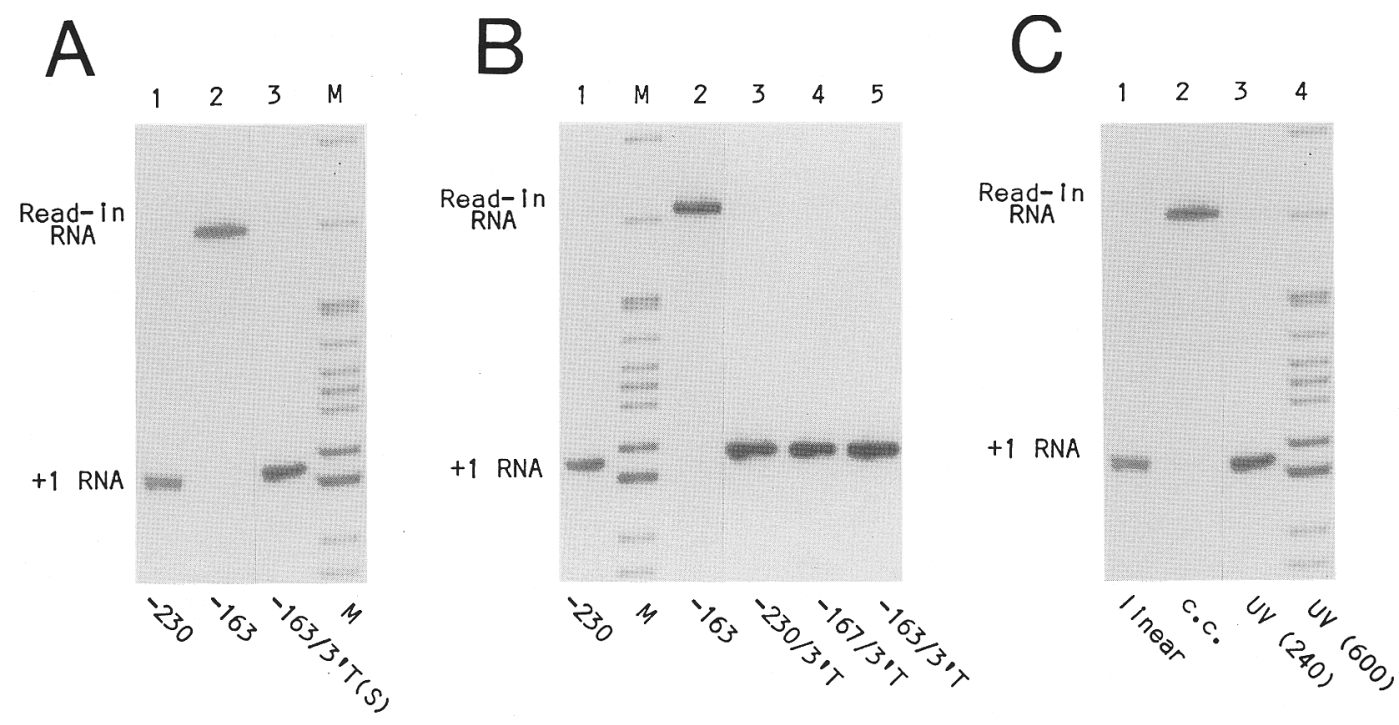

Figure 2. Stimulatory action of terminators distant from the natural promoter-proximal location. $(A$ and $B) 5^{\prime} \Delta-230,5^{\prime} \Delta-163$, and derivatives in which polymerase I terminator elements are inserted at either of two distant sites: $3^{\prime} \mathrm{T}(\mathrm{S}),(A)$; the $3^{\prime} \mathrm{T}$ series, $(B)$; were transiently transfected into cultured rodent cells. The amount of resultant +1 initiated RNA and read-in RNA was assessed by S1 nuclease mapping using a probe labeled at residue +155 . The transfection reactions all contained 1 pmole of rDNA plasmid per ml of media and the total DNA concentration was brought up to $3 \mu \mathrm{g} / \mathrm{ml}$ by addition of $\Delta \mathrm{pBR} . \mathrm{M}=\mathrm{HpaII}$ cleaved pBR322. (C) Equal amounts of variously treated $5^{\prime} \Delta-163$ templates were transcribed in vitro and the RNA products were assessed as in Fig. 1B. The templates were: (lane 1) linearized at the NdeI site, (lane 2) closed circular, (lanes 3 and 4) closed circular treated with UV light at 240 and 600 joules $/ \mathrm{m}^{2}$, repectively. 
Termination by $U V$-induced lesions also stimulates transcriptional initiation

To demonstrate that the transcriptional stimulation caused by the terminator element is due to its preventing read-around transcription and not to some unknown other property of this region, we have performed an analagous experiment in which transcription was efficiently terminated by an alternate mechanism. In this study (Fig. 2C) the closed circular $5^{\prime} \Delta-163$ template was treated with increasing doses of ultraviolet light to introduce lesions into the DNA that stop the passage of polymerase (Hackett and Sauerbier 1975). These templates were then transcribed in vitro and their products were assayed by $\mathrm{S} 1$ mapping. Confirming the results of Figure 1B, transcription of closed circular $5^{\prime} \Delta-163$ yields both correctly initiated and read-in RNA (Fig. 2C, lane 2). Yet templates treated with either 240 or 600 joules/ $\mathrm{m}^{2}$ of $254 \mathrm{~nm}$ (UV) light have acquired sufficient numbers of pyrimidine dimers to stop the polymerases before they transcribe completely around the plasmid and form read-in RNA (Fig. 2C, lane 3 and 4). Run-off transcription studies (not shown) demonstrate that the average lengths of the resultant transcripts are $\sim 1 \mathrm{~kb}$ and $\sim 0.5 \mathrm{~kb}$, respectively. Notably, however, both of these UV-treated closed circular templates direct appreciably more initiation (lanes 3 and 4) than does the control template that was not subjected to UV treatment (lane 2). In fact, they direct as much initiation as does a template in which read-around transcription was prevented by a different means-linearization of the template (lane 1). These data strongly suggest that inhibition of read-in transcription, whether by polymerase I termination elements, by UV-induced pyrimidine dimers, or by linearization of the template, significantly augments the level of transcriptional initiation relative to that obtained when polymerases read-around the entire plasmid molecule and into the promoter region from upstream.

\section{Read-in polymerases disrupt the association of} transcription factor $D$ with the promoter

As noted above, transcriptional interference (or promoter occlusion) could a priori occur either because closely packed polymerases that traverse a promoter block the attachment of new initiating polymerase molecules or because such read-in polymerases destabilize the transcription complex, thus preventing subsequent rounds of initiation. Since the terminator element can augment transcriptional initiation in the S-100 extract (Fig. 1B) where it is generally believed that the packing density of polymerases is far less than maximal, these polymerases seem unlikely to pose a steric hindrance to the attachment of additional polymerase molecules. We thus examined the alternative possibility that polymerases which transcribe through a promoter might destabilize its bound transcription factors.

We have found that two transcription factors are required for the expression of mouse rDNA: factor D which binds stably to the promoter and directs initia- tion, and factor $\mathrm{C}$ which is an activated subform of RNA polymerase I (Tower et al. 1986; Tower and SollnerWebb 1987). Earlier studies using linearized templates (on which polymerases should not read into the promoter region from upstream) have shown that factor D binds stably to the promoter in a preinitiation complex and that it remains attached to the promoter throughout the subsequent transcription period (Tower et al. 1986). Figure 3 shows experiments designed to investigate whether factor $\mathrm{D}$ is dislodged from its binding site on a promoter when transcription is allowed instead to proceed through that promoter, thereby testing whether the presumptive rDNA transcriptional interference is due to factor $\mathrm{D}$ destabilization. For this study we used a closed circular $5^{\prime} \Delta-163$ template to assure that there would be read-through transcription across the promoter (Henderson and Sollner-Webb 1986) and we then assayed if bound factor $\mathrm{D}$ would transfer to an added competitor template during a subsequent transcription period. Specifically, following preincubation of the $5^{\prime} \Delta-163$ template in the S-100 extract to allow formation of a stable preinitiation complex, a NdeI linearized competitor template was added and incubated for an additional 15 min. A 10-min period of transcription was then initiated by addition of rXTPs, during which time read-around transcription of the closed circular template could destabilize its bound factor D. Finally, the reaction was supplemented with $\left[\alpha{ }^{-32} \mathrm{P}\right] \mathrm{CTP}$ (to assess subsequent transcription) and with factor $\mathrm{C}$ (to allow detection of any transferred factor $D$, regardless of whether factor $C$ could also transfer), and transcription was allowed to proceed for $30 \mathrm{~min}$. As shown in the control reaction of Figure 3A, lane 3, when the first template is a Tth111I linearized molecule (which directs no read-around transcription), there is no transcription of the subsequently added NdeI linearized competitor template, and hence there is no transfer of factor D. (The mobility of the transcript from the NdeI linearized competitor template is shown in lanes 1 and 2 where this template is transcribed because it was present during the initial preincubation.) Strikingly, however, when the first template is closed circular, the subsequently added NdeI competitor template is also transcribed (lane 4). This demonstrates that the transfer to a competitor template occurs from a closed circular, but not from a linear, template molecule.

To confirm that transcription of the subsequently added template in the experiment of Figure 3A, lanes $3-5$ is due to the transfer of factor $D$, we performed the supplementation experiment shown in Figure 3A, lanes 6-9. The competitor template, which is hardly transcribed when added to a reaction that had preincubated with a linearized template (lane 6), is very efficiently transcribed when extra factor $D$ is added during the synthesis period (lane 8). Thus, transcription of the subsequently added competitor template is dependent on the availability of factor $\mathrm{D}$. The competitor template is also somewhat more actively transcribed in reactions that had preincubated with closed circular template when they are supplemented with additional factor D (lanes 7 

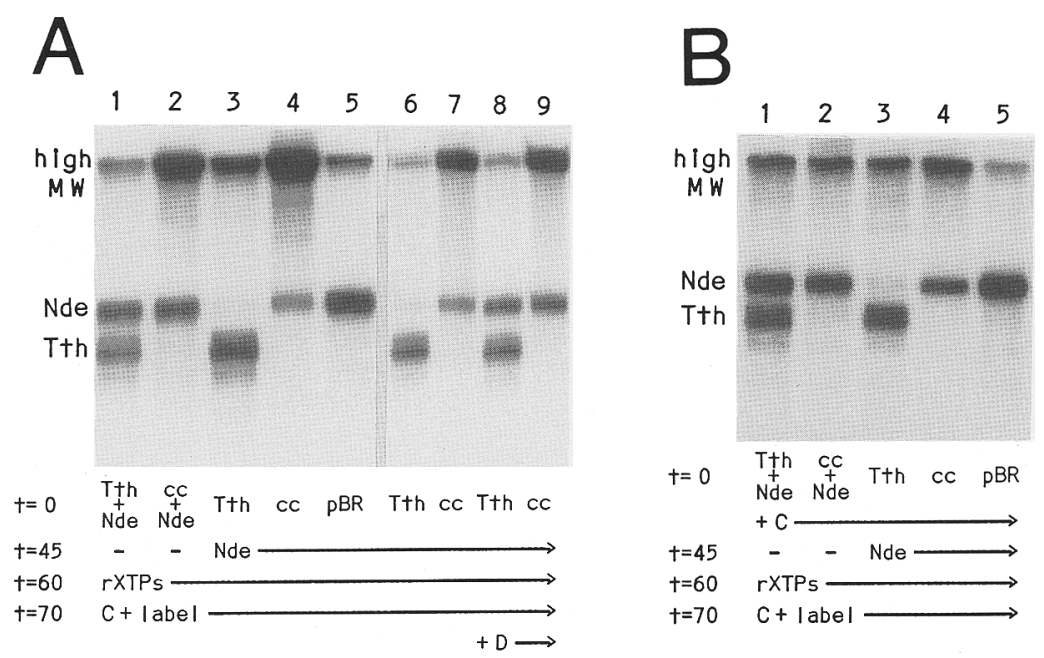

Figure 3. Displacement of factor $D$ by read-in transcription. $5^{\prime} \Delta-163$ linearized with Tth111I (lanes 1,3 ), closed circular $5^{\prime} \Delta$-163 (lanes 2, 4), or $\triangle \mathrm{pBR}$ (lane 5) was preincubated with S-100 extract factors in the absence of rXTPs for 0 (lanes 1,2) or 45 min (lanes 3-5) prior to the addition of a $5^{\prime} \Delta-163$ competitor template linearized with $N d e I$. At $60 \mathrm{~min}$, a 10 -min synthesis period was initiated by addition of rXTPs, and then factor $\mathrm{C}$ and $[\alpha-32 \mathrm{P} \mid \mathrm{CTP}$ were added for a 30-min labeling period. $(A)$ Lanes 6 and 7 and lanes 8 and 9 represent duplicate reactions to those of lanes 3 and 4 except that extra factor D was added to lanes 8 and 9 during the labeling period. Resultant radiolabeled RNA was resolved by electrophoresis and visualized by autoradiography. The high molecular weight RNA derives both from read-around transcription of the closed circular template and from end-to-end transcription of linear templates, as described in the text. $(A)$ The preincubation involved S-100 extract and, $(B)$ the preincubation involved the same extract supplemented with additional factor $\mathrm{C}$, the activated RNA polymerase $\mathrm{I}$.

and 91. The combined data of Figure 3A provides strong evidence that factor $\mathrm{D}$ is the component that is released from the closed circular template by transcription through the promoter.

In the experiment of Figure 3A, it appeared that approximately one third of the factor $\mathrm{D}$ transferred from the closed circular to the linear competitor template (compare lanes 2 and 4). This value, however, may be a significant underrepresentation of the maximal extent of $\mathrm{D}$ destabilization by polymerase read-in, because only those $\mathrm{D}$ molecules on templates that engaged an active polymerase would have been likely to transfer. Since our current extracts contain a vast excess of $D$ activity over $\mathrm{C}$ activity (data not shown), we reasoned that a large fraction of the $\mathrm{D}$ molecules might not have had the opportunity to transfer prior to the onset of the labeling period (when the distribution of factor $D$ is assessed in the presence of excess active polymerase). To circumvent this limitation, we repeated the experiment of Figure 3A with an S-100 extract supplemented with additional factor $\mathrm{C}$ to allow substantially more transcription during the initial synthesis period. As shown in Figure 3B, the additional factor $C$ does not reduce the stability of the transcription complex formed on a linear template (lane 3). Yet now comparable amounts of transcript are obtained from the NdeI linearized competitor template whether it is added to an extract that had preincubated with the closed circular template (lane 4) or added simultaneously with the closed circular template (lane 2). The transfer of factor D from its original binding site on a promoter that experiences polymerase read-in to a linear template is thus shown to be quite efficient. Comparison of the data of Figures 3A and 3B also provides further evidence that the transfer process is transcription dependent.

While read-around transcription of closed circular templates yields transcripts that migrate near the top of the gel (as in Fig. 3) such high moleular weight RNA can also result from nonspecific end-to-end transcription (Kurl and Jacob 1985). The read-around transcripts from the preincubated closed circular template are readily apparent in experiments performed as in Figure 3A. However, when the preincubations are supplemented with extra factor $\mathrm{C}$ (as in Fig. 3B), much of the factor D has already transferred from its original binding site on the closed circular template to the linear competitor template during the initial transcription period, and thus only limited amounts of read-around RNA are transcribed during the labeling period. Such initial readaround RNA can readily be detected by performing the labeling throughout the entire transcription period (data not shown).

The conclusion that factor $D$ is displaced from its binding site in a promoter by transcription through that promoter, is dependent on the factor D having stably associated with the first template during the prebinding period, prior to the addition of the competitor template. This assertion was validated in the control reactions shown in Figure 3, lanes 3, where factor D did not transfer to a subsequently added competitor template when it had been preincubated with a linearized plasmid that should direct no read-in transcription; this same stable binding of factor $\mathrm{D}$ is observed both in the absence (Fig. 3A) and in the presence (Fig. 3B) of excess factor C. 
Nonetheless, to rigorously prove that a factor $\mathrm{D} / \mathrm{rDNA}$ stable preinitiation complex also forms on a closed circular template, the experiment of Figure 4 was performed. In this study, a closed circular or linearized template was preincubated in the S-100 extract and then a linearized competitor template was added and incubated, as in the experiment of Figure 3A. Next, the closed circular template was linearized (by treatment with PstI) to prevent polymerases from traversing its promoter and liberating its bound factor D. Finally, as in the experiment of Figure 3, after a period of transcription, factor $C$ and $\left[\alpha^{-32}\right.$ P]CTP were added for an additional $30 \mathrm{~min}$ to assay for transfer of factor $\mathrm{D}$. As shown in Figure 4A, lane 2, cleavage of the closed circular template after it has bound to factor $\mathrm{D}$, but before it has initiated transcription, prevents the transfer of factor $\mathrm{D}$ (compare to lane 4). Thus factor D binds stably to a closed circular template and does not transfer to another DNA molecule either during an incubation in the absence of transcription or during the transcription process after the template had been linearized.

Another important control is to demonstrate that factor D does not simply get dislodged from the closed circular template during the transcriptional elongation process, due to topological effects particular to a closed circular template. We have addressed this issue by two complementary experiments. The first study has shown that when transcription on a closed circular template is stopped before reading completely around the plasmid and into the initiation region, the bound factor $D$ is not similarly liberated. This experiment used the same UVtreated $5^{\prime} \Delta-163$ DNA preparation that was shown in Figure 2C to support large amounts of transcriptional initiation but minimal amounts of read-in transcription. With this template (treated at 240 joules $/ \mathrm{m}^{2}$ ), UV-induced lesions stop the elongating polymerases after they have read, on the average, about one third of the way around the plasmid (data not shown). When the transfer protocol of Figure 3 is repeated by preincubating with this closed circular UV-treated DNA, the transcription of the competitor template is very greatly reduced (Fig. $4 \mathrm{~B}$, lane 2), relative to that which occurs in parallel control reactions with untreated closed circular template (Fig. 4B, lane 1). Thus, the release of factor D from a closed circular template is not due to the process of transcription per se, but requires that polymerases proceed completely around the template molecule.

We have also addressed the topology issue by an independent experiment, in which transcription is allowed to proceed completely around a closed circular template under conditions where the template is topologically relaxed. Specifically, reactions analogous to those of Figure 3 were supplemented with large amounts of topoisomerase $\mathrm{I}(400 \mathrm{U} / \mathrm{ml})$ during the transcription period
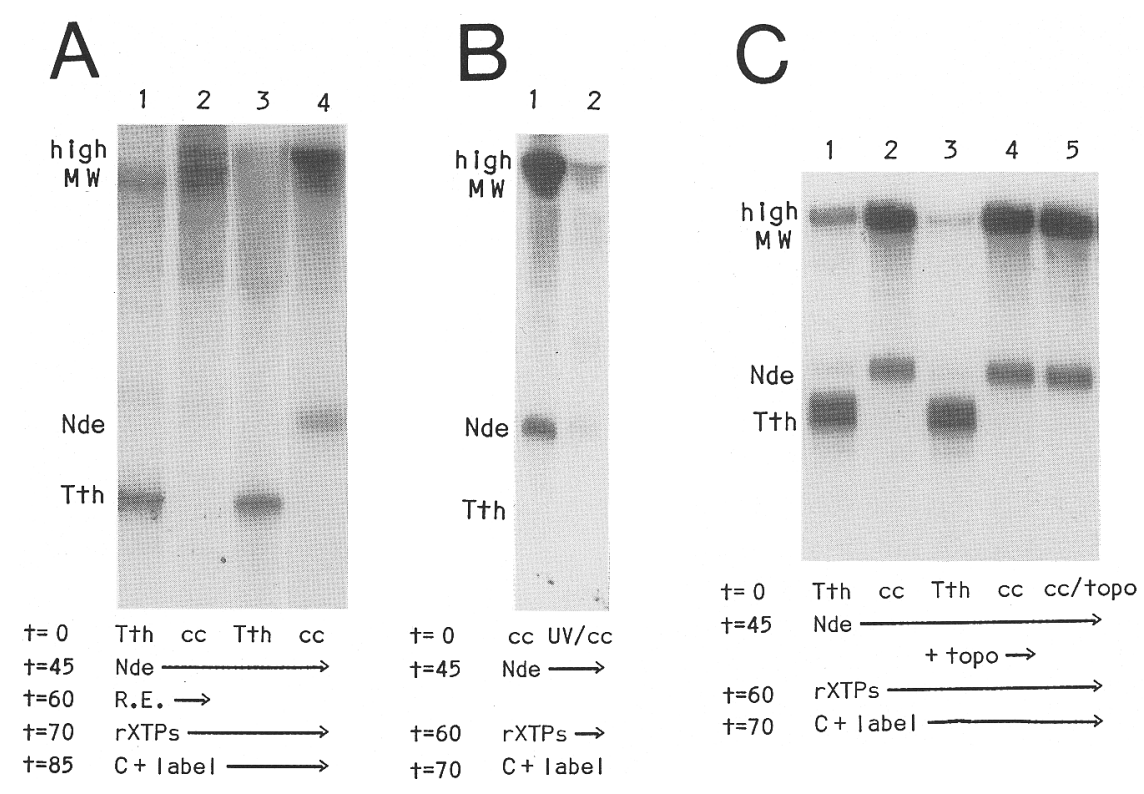

Figure 4. Factor D binds stably to a closed circular template in the absence of read-in transcription. $(A)$ Following a 45-min preincubation of Tth 111 linearized 5' $\Delta$-163 (lanes 1,3) or closed circular 5' $\Delta-163$ (lanes 2, 4) in the S-100 extract, NdeI linearized 5' $\Delta$-163 was added for an additional 15-min incubation in the absence of rXTPs (as in Fig. 3A). The assembled templates in lanes 1 and 2 were then cleaved by a 10 -min treatment with the restriction enzyme (R.E.) PstI which cuts $1.8 \mathrm{~kb}$ downstream of the initiation site. Next, rXTPs were added for $15 \mathrm{~min}$, after which time factor $\mathrm{C}$ and $\left[\alpha^{-32} \mathrm{P}\right] \mathrm{CTP}$ were added for a 30 -min labeling period. Transcripts were analyzed as in Fig. 3. $(B)$ Transcriptions were performed as in $A$, lane 4; preincubating with: (lane 1) closed circular $5^{\prime} \Delta-163$ or (lane 2) closed circular $5^{\prime} \Delta-163$ that had been treated with 240 joules $/ \mathrm{m}^{2}$ of UV light (the same DNA preparation that was used in Fig. $2 \mathrm{C}$, lane 3). The heterogeneously sized transcripts from the UV-treated template are not visible at this exposure because they are dispersed throughout the gel lane. (C) Transcriptions were performed as in $A$, lanes 3 and 4 , using the indicated closed circular or linearized templates. In lanes 3 and $4,400 \mathrm{U} / \mathrm{ml}$ of Topo I was added during the labeling period; in lane 5, prior to the start of the reaction the DNA was relaxed with Topo I, which remained present in the reaction at $25 \mathrm{U} / \mathrm{ml}$. 
(Fig. 4C, lane 3 and 4) or they were formed using topoisomerase I-relaxed DNA and the enzyme remained present during the entire reaction time course (lane 5). Control experiments (not shown) demonstrated that fully supercoiled DNA is relaxed with a $t_{1 / 2}$ of $\sim 15 \mathrm{sec}$ in these topoisomerase I-supplemented reactions. Yet the same amount of factor $D$ is released in the reactions containing relaxed templates (lanes 4 and 5) as in the control reaction (lane 2). This experiment shows that transcription around a closed circular template causes the release of its bound factor $D$, even when the template is maintained in a topologically relaxed state.

The aggregate data of Figure 3 and 4 demonstrate that: (1) factor $D$ binds stably to a closed circular template in a preinitiation complex; $(2)$ factor $D$ is not released by the process of transcription per se; (3) factor $D$ is released when transcription proceeds completely around the plasmid molecule, even when the plasmid remains relaxed. This provides very strong evidence that factor $D$ is released (or 'snowplowed') from its binding site in the rDNA promoter when transcription proceeds across that promoter region from upstream.

\section{Factor $C$ is not similarly dislodged}

When the passage of a transcribing polymerase destabilizes the factor $\mathrm{D} / \mathrm{rDNA}$ interaction, one might anticipate that only the factor $\mathrm{D}$ would transfer to a competitor template and that the active polymerase itself would not be displaced. The data of Figure 5 confirm this hypothesis. In this experiment, the transfer protocol of Figure $3 \mathrm{~A}$ was repeated except that additional factor $\mathrm{C}$ was not added in the labeling period. In this case, there was only a very low amount of transcription of the competitor template (lane 2) indicating that initiation-competent polymerase did not transfer along with the factor $D$. Furthermore, when this experiment was repeated with extra factor $\mathrm{D}$ added after the preincubation period, there was no appreciable increase in the amount of competitor transcription (data not shown), providing additional evidence that significant amounts of factor $\mathrm{C}$ are not transferred. Nonetheless, in the control reaction where factor $\mathrm{C}$ was added, there was the usual large amount of competitor transcription due to transfer of factor $\mathrm{D}$ (lane 5; see lighter exposure in lane $5^{\prime}$ to directly compare with lane 2 ). We thus conclude that factor $C$ is not released from the template in a functional form when polymerases transcribe through and destabilize the factor D-promoter complex.

It might be noted that these data are fully compatible with our previous demonstration that transcription of a closed circular template does not cause the release of both its bound factors D and C (Tower et al. 1986). Indeed, the current data extend the previous results by showing that $D$ but not $C$ is released by transcription around a closed circular template (Figs. 4 and 5), but that $D$ remains stably bound during transcription of a linear template (Fig. 4).
The release of factor $D$ is a time-dependent process

Our understanding of the process by which factor $\mathrm{D}$ is destabilized during transcription on a closed circular template predicts that the $\mathrm{D}$ release would be a time-dependent process. The transfer of factor $D$ to the competitor template should begin only after the first polymerases have read around the entire plasmid molecule and entered the promoter region from upstream, and could continue as increasing numbers of the closed circular templates are thus transcribed. At an elongation rate of 25-30 nucleotides per sec (Cavanaugh and Thompson 1985; J. Tower and B. Sollner-Webb, unpubl.) polymerases traverse the closed circular $5^{\prime} \Delta-163$ template in $\sim 1.5 \mathrm{~min}$, so the competitor template should only start being transcribed $\sim 1.5$ min after transcription is initiated by addition of rXTPs to the reaction. To assess this prediction, the experiment of Figure 6 was performed. Closed circular 5' $\Delta-163$ (Fig. 6A, lanes 1-3), linear $5^{\prime} \Delta-163$ (lanes 4-6), or a nonspecific DNA (lanes $7-9$ ) was incubated in the S-100 extract prior to addition of the NdeI-cleaved tester template. Transcription was then initiated by addition of rXTPs, and 0,1 , or $4.5 \mathrm{~min}$ later, a $1.5-\mathrm{min}$ labeling period was begun in the presence of additional factor C. Transcription of the template to which the factor $\mathrm{D}$ had been bound during the preincubation is maximal in the first $1.5 \mathrm{~min}$ following the addition of rXTPs (lanes 4 and 7) and then slowly decreases (lanes 5, 6 and 8,9), consistent with our previous observations showing a burst of transcription from a preincubated template upon addition of IXTPs (V. Culotta and B. Sollner-Webb, unpubl.). In contrast, in the reaction in which factor $D$ had initially been bound to the closed circular 5' $\Delta-163$ template, transcription of the competitor template exhibits a strikingly different time course. It only begins at $\sim 1.5 \mathrm{~min}$ (lane 1 ) and then continues to increase in rate over the next $\geqslant 4.5 \mathrm{~min}$ (lanes 2,31 . The time course data from Figure $6 \mathrm{~A}$ and from several duplicate experiments have been quantitated by

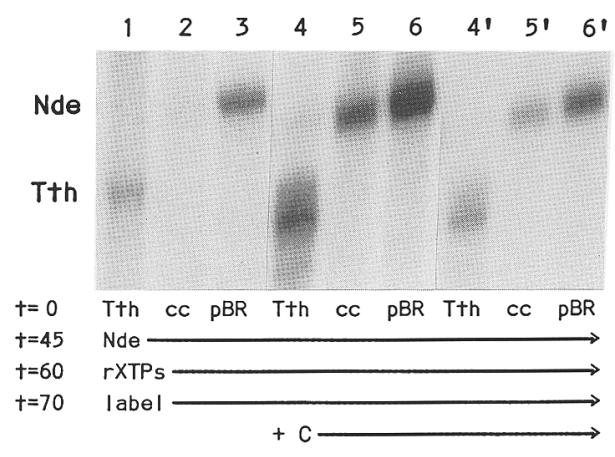

Figure 5. Factor $\mathrm{C}$ is not displaced upon transcription of a closed circular template. Reactions were performed as in lanes 3-5 of Fig. 3A except that no factor $\mathrm{C}$ was added to the reactions in lanes 1-3 during the labeling period. Lanes $4^{\prime}-6^{\prime}$ are a lighter exposure of lanes 4-6 to facilitate comparison with lanes 1-3. 

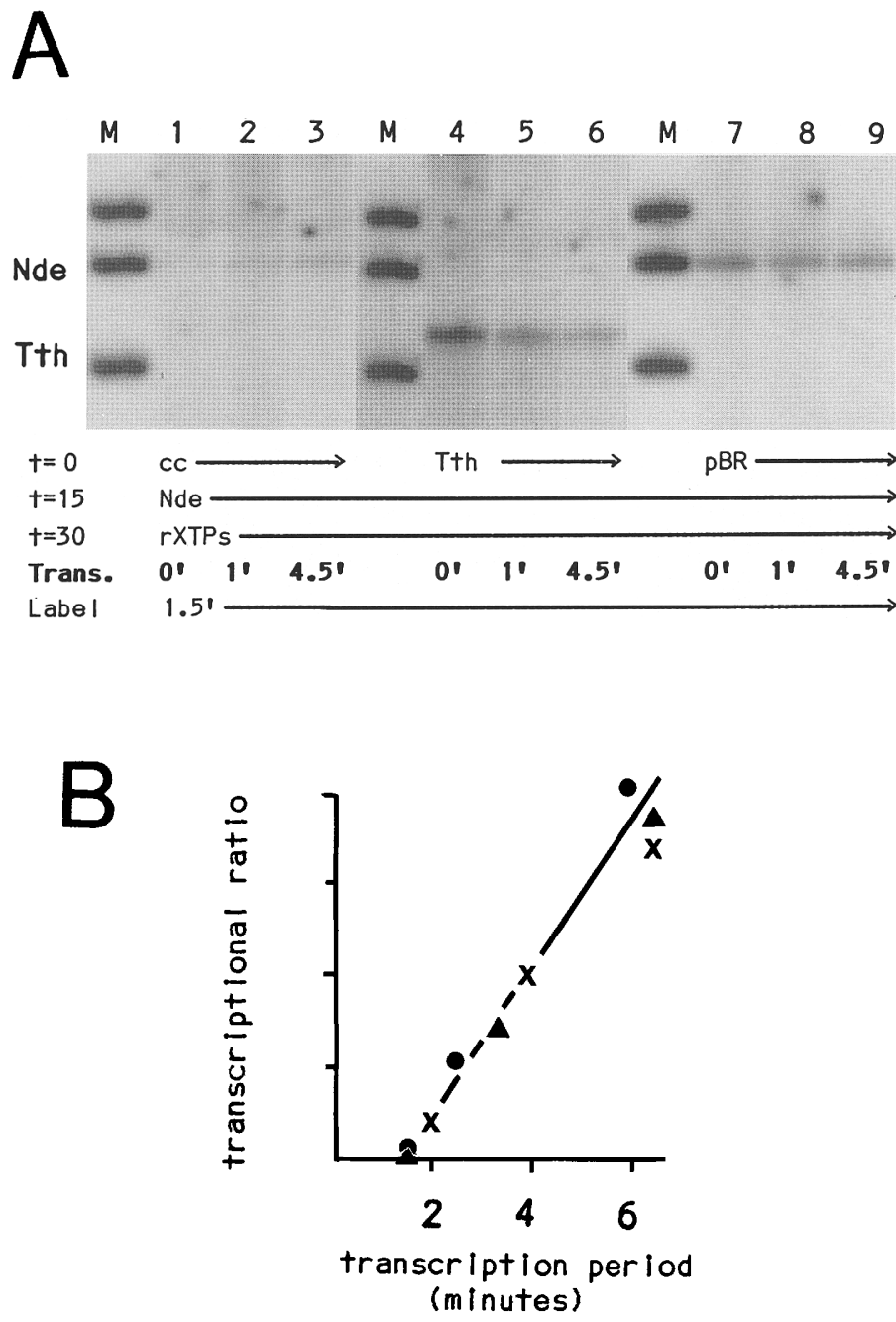

densitometric scanning of the autoradiograms. These results are most readily compared by plotting, for each time point, the ratio of the amount of transcript obtained from the competitor template as in lanes 1-3 of Fig. 6A) to that from the preincubated template (as in lanes $4-6)$. Figure $6 \mathrm{~B}$ shows that this ratio increases linearly with time, and the line that best fits the data intersects the abscissa at $\sim 1.5 \mathrm{~min}$, indicating that transfer in these various experiments begins at $\sim 1.5 \mathrm{~min}$. These data demonstrate that factor $D$ is released from the closed circular template and rebinds to the competitor template with the kinetics predicted if the release is caused by polymerases that transcribed completely around the closed circular template.

\section{Discussion}

Earlier work has demonstrated the existence of transcriptional terminators immediately upstream of rodent rDNA promoters (Grummt et al. 1986; Henderson and Sollner-Webb 1986), and these terminators have the striking property of greatly augmenting polymerase I
Figure 6. Kinetics of the displacement of factor D. $|A|$ Closed circular 5' $\Delta$-163 (lanes 1-3), 5' $\Delta$-163 linearized with Tth 111 I (lanes $4-6$ ), or $\Delta$ pBR (lanes 7-9) were preincubated in the S-100 extract for 15 min prior to addition of $N d e I$ linearized $5^{\prime} \Delta-163$. After an additional $15-\mathrm{min}$ incubation, rXTPs and an aliquot of factor $\mathrm{C}$ was added. Zero, 1 , or $4.5 \mathrm{~min}$ later, $\left[\alpha^{-32} \mathrm{P}\right] \mathrm{CTP}$ and additional factor $\mathrm{C}$ were added to initiate a 90 -sec labeling period. RNA was analyzed as in Fig. 3. $\mathrm{M}=H$ paII cleaved pBR322. After an extended preincubation there is a rapid burst of transcription of the preincubation template complex, in accordance with our previous observations. Only subsequently does the relative amount of transferred factor D/reflected in the relative amount of competitor transcription/ become extensive (as seen in Figs. 3-5 and in Fig. 6A lanes 6 versus 3). (B) Data from Fig. 6A $(\bullet)$ and two other independent kinetic experiments $(\boldsymbol{\Lambda}, X)$ were quantitated by densitometric scanning. On the ordinate is plotted the ratio of the intensity of the competitor transcript in reactions preincubated with closed circular template (as in Fig. 6A, lanes $1-3)$ to the intensity of the transcript from the preincubated linear template in reactions as in lanes 4-6. The full scale is 0.4 for the experiments depicted by the solid symbols and 0.2 for the one shown by the $X$. The abscissa represents the time, in min, of the total transcription period; the labeling period comprised the last $90 \mathrm{sec} .(\bullet, \mathbf{\Delta})$ or $120 \sec (x)$.

transcription from the adjacent rDNA promoter (Henderson and Sollner-Webb 1986). In transiently transfected cells the transcriptional stimulation is one to two orders of magnitude, and a similar stimulatory effect, but of a smaller magnitude, is also observed in vitro with closed circular transcription templates (e.g., Fig. 1). We have entertained several models for how this transcriptional stimulation may occur:

1. The terminator could efficiently 'hand-off' polymerases to the adjacent promoter in a concerted reaction, the 'read-through enhancement' model (Moss 1983; Henderson and Sollner-Webb 1986; McStay and Reeder 1986; Mitchelson and Moss 1987).

2. The terminator could actually be a promoter element or a polymerase I enhancer, with the termination effect being a mere consequence of transcribing polymerases running into a 'brick wall' created by the bound transcription factor (Baker and Platt 1986). Alternatively, this rDNA region could encode both a terminator and an independent promoter or enhancer element (McStay and Reeder 1986). Consistent with this class of models, 3 ' end formation directed by the -168 sequence is known to be dependent on the binding of a trans- 
acting factor (Henderson and Sollner-Webb 1986; Bartsch et al. 1988).

3. Alternatively, the terminator could serve to protect the initiation process from damage that might otherwise be caused when approaching polymerases transcribe across that promoter. Such damage could be due to the read-through polymerases sterically blocking attachment of new polymerases (Adhya and Gottesman 1982; Cullen et al. 1984; Proudfoot 1986) or to their disrupting the otherwise stable binding of a transcription factor to the promoter.

In the studies described in this paper, we experimentally assess these models. Scenarios involving readthrough enhancement (model 1 ) or envisioning that the terminator is or overlaps a promoter domain (model 2) would demand that the transcriptional stimulatory effect of the terminator be dependent on its proximity to the promoter. Yet the experiments of Figure 2A and B show that polymerase I terminators act in a position-independent manner, for they stimulate transcription at least as well when located $3.7 \mathrm{~kb}$ upstream from the initiation site as when at the normal promoter-proximal position. Furthermore, other methods of preventing readaround transcription, such as introducing UV lesions or linearizing the template DNA (Fig. 2C), similarly stimulate initiation. The fact that the promoter-proximal terminator element has no effect on the level of transcription from templates where polymerases are stopped before reading up to this region (Fig. 2B) provides further evidence that the mouse rDNA terminators are not functioning as promoter elements. Also, the inability of the terminator to act in the reverse orientation (Henderson and Sollner-Webb 1986) suggests that it is not an enhancer element.

Models 1 and 2 being thus disfavored, we turned our attention to model 3 in which the terminator protects against transcriptional interference. Since the terminator element stimulates transcription on closed circular templates in vitro where polymerase density is generally thought to be fairly low, it seemed unlikely that it acts by relieving a steric impediment to polymerase attachment caused by closely packed traversing polymerases. The experiments of Figures 3-5 show the veracity of the final possibility raised above in model 3 - that otherwise stably bound transcription factors are displaced by transcription through the promoter complex. Specifically, we demonstrate that factor $D$, the essential sequence-specific, promoter-binding transcription factor, is displaced from the template by polymerase passage (Fig. 3). These released D molecules are then able to productively attach to another rDNA molecule from which they can direct further rounds of transcriptional initiation. Factor $\mathrm{C}$ is not similarly released in an active form (Fig. 5).

Controls affirm that the release of factor $D$ requires transcription of the template to which the $D$ was originally bound but that it is not transcription per se that causes the destabilization, for factor $\mathrm{D}$ remains stably bound to closed circular templates on which readaround transcription has been inhibited by UV treat- ment as well as to linear templates that are being transcribed (Figs. 3; 4A,B). The release of factor D also cannot be attributed to supercoils that accumulate throughout the transcription process (Fig. 4C). Instead, the release of factor $\mathrm{D}$ is dependent on transcription reading into the promoter region from upstream, with the traversing polymerases evidently acting like a 'snow-

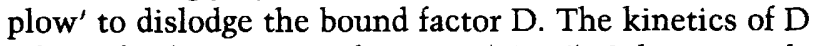
release further support this view (Fig. 6). Other controls demonstrate that factor $\mathrm{D}$ does indeed bind in a stable preinitiation complex to closed circular as well as to linear templates (Fig. 4A) and that the release of factor D is actually what is being assessed (Fig. 3A). It might also be mentioned that the choice of the plasmid vector is likely to be important in these experiments, for the EcoRI-PvuII region of pBR322, which is deleted in our constructs, directs fortuitous polymerase I initiations [Culotta 1987) which could interfere in experiments involving read-in transcription. Indeed, such vector initiations could provide an alternate explanation for data that was interpreted to indicate that the terminator element stimulated initiation on linear templates where there was no intentional read-in transcription (Grummt et al. 1986).

Another important conclusion from the data in this paper is that a single template molecule serves to direct many rounds of transcription, both in the S-100 extract and in transfected cells. Were this not the case, and only one transcript was produced from each transcription complex, disruption of these complexes by passage of the polymerase would not decrease the level of transcription, contrary to the data of Figure 1. Prior to these results, evidence for reinitiation occurring on cloned rDNA templates in vitro was derived solely from calculated values of $\leqslant 4$ RNAs produced per input rDNA molecule (Cizewski and Sollner-Webb 1983; Wandelt and Grummt 1983). Even though this number may be artificially low (since probably not all the rDNA molecules in the reaction direct production of RNA), it is too close to 1 to have provided unequivocal evidence for reinitiation.

It is noteworthy that disruption of the stable transcription complex caused by the passage of one or only a few polymerases can prevent a potentially large number of subsequent initiation events at that promoter. This understanding provides an explanation for what initially seemed a surprising result, namely, that the transfected $5^{\prime} \Delta-230$ template directs the accumulation of less RNA than does $5^{\prime} \Delta-230 / 3^{\prime} \mathrm{T}$ (which has the same promoter and adjacent terminator as $5^{\prime} \Delta-230$ but also contains additional polymerase I terminators interposed at the opposite side of the plasmid; Fig. 2B). If termination on the transfected $5^{\prime} \Delta-230$ template (with only a promoterproximal terminator) is not as efficient as that on a plasmid which also contains multiple $3^{\prime}$ terminators, then the small fraction of read-in transcripts that occur on $5^{\prime} \Delta-230$ could disrupt a significant fraction of promoter complexes and thereby lower the level of initiation.

A recent study by Bateman and Paule (1988) has demonstrated that RNA polymerase I transcription across 
the rDNA promoter of Acanthamoeba releases its bound transcription initiation factor TIF. Because polymerase I read-in causes an analogous disruption of stable transcription complexes in organisms as diverse as mouse and protist, this is likely to be a general effect of transcription through rDNA promoters. This implies that the promoter-proximal polymerase I terminators found in the rDNA of many metazoan species will all serve to protect their transcription factor-promoter complexes.

The disruption of the rDNA stable transcription complex caused by polymerase I read-in may be compared to a small number of examples of transcriptional interference in other systems. The phenomenon of promoter occlusion was first detected with prokaryotic trp and gal promoters (Hausler and Somerville 1979; Adhya and Gottesman 1982) where it was concluded that closely packed polymerases reading through a promoter inhibit subsequent initiation at that promoter by sterically blocking attachment of new polymerase molecules. Evidently most other prokaryotic promoters do not initiate at a high enough rate to exert this effect (Adhya and Gottesman 1982). Transcriptional interference has also been documented for transfected eukaryotic promoters that are transcribed by RNA polymerase II, both with natural tandem promoters (Cullen et al. 1984) and with artificial tandem constructs (Kadesch and Berg 1986; Proudfoot 1986). Although it has been proposed that this transcriptional interference was also due to a steric blockage to the attachment of new polymerase molecules (Proudfoot 1986), we speculate that it might alternatively arise from a disruption in the binding of promoter-stimulatory factors, in analogy with the situation described in this paper for polymerase I promoters. In the cases of transcriptional repression of downstream polymerase II promoters noted above, the effects are 3 to 5-fold, but this most likely represents a minimum value; in order for such transcriptional interference to be observed, both the upstream and the downstream promoters of the same transfected template molecule would need to be activated for transcription.

In standard metazoan in vitro transcription systems, such transcriptional interference on tandem promoters seems relatively unlikely to be observed, since only a small fraction of the input template molecules direct transcription. Indeed, from such in vitro transcription studies of constructs bearing tandem mouse rDNA promoters it has been concluded that polymerase I promoters are not subject to transcriptional interference since the downstream and upstream promoters exhibited similar levels of transcription (Grummt et al. 1986). We suggest that this observed lack of transcriptional interference is not contrary to the results presented in this paper, but rather that the two tandem rDNA promoters were both active on only a small fraction of the in vitro template molecules. In contrast, our current in vitro experimental protocol is able to demonstrate that rDNA promoters are sensitive to transcriptional interference because the same promoter whose transcription is being assessed also provides the read-in polymerase.
It remains to be determined whether stopping of polymerases that would otherwise disrupt the stable transcription complex is the only mechanism by which the mammalian promoter-proximal terminator stimulates rDNA transcription. This is certainly its major effect in our assays, and it is also likely to be quite significant in vivo on the endogenous mammalian rRNA genes because polymerases clearly do approach the initiation region from upstream. These spacer polymerases include ones that initiated at promoters in the rDNA spacer (Cassidy et al. 1987; Harrington and Chikaraishi 1987; Kuhn and Grummt 1987; Tower et al. 1989) and possibly also ones that failed to terminate at the $3^{\prime}$ end of the previous tandem rRNA gene. A terminator element could, however, have other positive effects on transcription as well. In particular, the possibility of read-through enhancement remains intriguing. On the one hand, using Xenopus rDNA plasmids analogous to our 3'T series, Mitchelson and Moss (1987) reported that the level of initiation is depressed relative to that of the parental plasmid lacking the inserted terminators, consistent with a read-through enhancement mechanism. On the other hand, data from UV inactivation studies (Hackett and Sauerbier 1975 and Fig. 2C above; Johnson et al. 1987; P. Labhart and R. Reeder, pers. comm.) suggest that polymerase hand-off is not a major factor in maintaining the high level of either mammalian, trypanosomatid, or frog rDNA initiation, since lesions that prevent polymerases from progressing through the gene do not significantly alter the level of initiation. Furthermore, in some species' rDNA, promoter elements could also overlap or reside within the promoter-proximal terminator sequence (McStay and Reeder 1986; C. Pikaard and R. Reeder, pers. comm.). Future work will be needed to determine whether the terminator elements in different species actually exhibit different mechanisms of stimulation or whether alternate interpretations can unify the existing data into one cohesive model for the action of promoter-proximal rDNA terminators.

\section{Methods \\ Plasmid templates}

Plasmid templates $5^{\prime} \Delta-230,5^{\prime} \Delta-168,5^{\prime} \Delta-167$, and $5^{\prime} \Delta-163$ contain mouse rDNA from the indicated $5^{\prime}$ position to residue +292 , inserted between the EcoRI and PvuII sites of pBR322, as previously described (Miller et al. 1985; Henderson and SollnerWebb, 1986). From these plasmids the 3'T series was created by inserting an $\sim 1.9-\mathrm{kb}$ SmaI-NdeI fragment containing all 8 of the natural rRNA gene terminator elements (as defined in Grummt et al. 1985) between the PvuII and NdeI sites of the pBR322 region. $5^{\prime} \Delta-163 / 3^{\prime} T(S)$ was prepared by inserting this $3^{\prime}$ termination region between the SmaI site at rDNA residue +155 and the $\mathrm{pBR} 322 \mathrm{NdeI}$ site. $\triangle \mathrm{pBR}$ is a derivative of $\mathrm{pBR} 322$ from which the EcoRI-NdeI region containing the tet gene was deleted. To prepare the $5^{\prime} \Delta-163$ template with UV lesions, this DNA [75 $\mu \mathrm{g} / \mathrm{ml}$ in $10 \mathrm{~mm}$ Tris- $\mathrm{HCl}$ (pH 7.5), $0.1 \mathrm{mM}$ EDTA] was irradiated on a rotary shaker at a depth of $1-2 \mathrm{~mm}$ in a plastic microwell plate. The irradiation was performed using a General Electric germicidal lamp (254 nm peak) for varying times at 2 joules $/ \mathrm{m}^{2} / \mathrm{sec}$. The dose was measured with a BlakRay UV meter, model 225 . The integrity of the templates was 
confirmed by agarose gel electrophoresis and the abundance of UV lesions that stop transcription was determined from the length distribution of radiolabeled RNAs transcribed from these templates.

\section{Analysis of transcription in transiently transfected cells}

Plasmids were transfected into growing $\mathrm{CHO}$ cells using the DEAE dextran:DMSO protocol (Lopata et al. 1984), and RNA was isolated by the guanidine: $\mathrm{CsCl}$ procedure (Chirgwin et al. 1979), as detailed in Henderson and Sollner-Webb (1986). S1 mapping analysis of these RNAs utilized a probe prepared from rDNA plasmid p5' Sal-Pvu (Miller and Sollner-Webb 1981), 5'end labeled at residue +155 or +292 and made single stranded by Exonuclease III digestion, as described (Henderson and Sollner-Webb 1986). All results were confirmed in, at least, duplicate or triplicate sets of independent transfection experiments, which yielded virtually identical data to these shown. [It has not proven possible to use neutral noncompeting internal controls for such in vivo rDNA expression studies because added marked rRNA genes compete with the rRNA gene under study which can significantly affect results. Furthermore, transcription catalyzed by polymerase II (tk gene) and polymerase III ( $5 \mathrm{~S}$ gene) has a different response to varying template concentrations than does a polymerase I promoter (data not shown), and thus these templates are not useful control genes either.]

\section{In vitro transcriptions}

In vitro preincubation and transcription reactions were conducted basically as described (Tower et al. 1986) except that the $25 \mu \mathrm{l}$ reactions contained $2 \mu \mathrm{l}$ of mouse cell S-100 extract, 150 ng of the plasmid carrying the test template, and $250 \mathrm{ng}$ of the plasmid bearing the NdeI linearized template. For most experiments, we have used a $5^{\prime} \Delta-163$ deletion template which contains all of the identified polymerase I promoter elements (residues approximately -140 to +5 ; Miller et al. 1985) but lacks the -168 terminator element which could direct the release of incoming polymerases prior to their traversing the promoter. The precise order of additions and the incubation times are given in the respective figure legends. Where indicated, the preincubated template was cleaved by treatment with $10 \mathrm{U}$ of PstI (BRL) for $10 \mathrm{~min}$ at $30^{\circ} \mathrm{C}$. In some reactions, the template was relaxed with calf thymus topoisomerase I (BRL) prior to the preincubation with extract or topoisomerase I was added before the start of the transcription period, as indicated in the figure. In most reactions, factor $\mathrm{C}$ was also added during the labeling period. The $C$ activity was prepared by passage of the S-100 extracts over phosphocellulose P-11 where it elutes between 0.35 and $0.6 \mathrm{M} \mathrm{KCl}$. It was brought to $0.1 \mathrm{M} \mathrm{KCl}, 5 \mathrm{mM} \mathrm{MgCl} 2,20$ mM HEPES (pH 7.9), $1 \mathrm{~mm}$ DTT, $0.1 \mathrm{~mm}$ EDTA, $2 \mu \mathrm{M}$ leupeptin, $0.5 \mu \mathrm{M}$ pepstatin A, $20 \%$ glycerol by dialysis and exhibits a high activity, devoid of any detectable D activity. Isolation and electrophoretic analysis of the in vitro transcribed, radiolabeled RNA was described (Miller and Sollner-Webb 1981). The transcription reactions of Figure 1B were performed as described in Henderson and Sollner-Webb (1986). All in vitro transcription results were confirmed in multiple independent transcription reactions, only one representative example of which is reproduced for the figures.

\section{Acknowledgments}

We thank Dr. David Schlessinger for providing 'plasmid 3'-end RI-RI' from which the rRNA 3' terminator segment was iso- lated and Drs. Ron Reeder, Craig Pikaard, Paul Labhart and Eric Bateman for communicating results prior to publication. We also thank Drs. Ed Mougey, Susan Kass, Louise Pape, and John Tower for helpful discussions. We gratefully acknowledge the technical assistance of Ms. Maria Isern, and Ms. Margaret Minnick's help in preparing the manuscript. This work was supported by grant GM27720 from the NIH.

\section{References}

Adhya, S. and M. Gottesman. 1982. Promoter occlusion: transcription through a promoter may inhibit its activity. Cell 29: 939-944.

Baker, S.M. and T. Platt. 1986. Pol I transcription: which comes first, the end or the beginning? Cell 47: 839-840.

Bartsch, I., C. Schoneberg, and I. Grummt. 1988. Purification and characterization of TTFl, a factor that mediates termination of mouse rDNA transcription. Mol. Cell. Biol. 8: 3891-3897.

Bateman, E. and M. Paule. 1988. Promoter occlusion during rRNA transcription. Cell 54: 985-992.

Cassidy, B.G., H.-F. Yang-Yen, and L.I. Rothblum. 1987. Additional RNA polymerase I initiation site within the nontranscribed spacer region of the rat rRNA gene. Mol. Cell. Biol. 7: 2388-2396.

Cavanaugh, A.H. and E.A. Thompson, Jr. 1985. Hormonal regulation of transcription of rDNA: glucocorticoid effects upon initiation and elongation in vitro. Nucleic Acids Res. 13: 3357-3369.

Chirgwin, J.M., A.E. Przybyla, R.J. MacDonald, and W.J. Rutter. 1979. Isolation of biologically active ribonucleic acid from sources enriched in ribonuclease. Biochemistry 18: 52945299.

Cizewski, V. and B. Sollner-Webb. 1983. A stable trancription complex directs mouse ribosomal RNA synthesis by RNA polymerase I. Nucleic Acids Res. 11: 7043-7056.

Cullen, B.R., P.T. Lomedico, and G. Ju. 1984. Transcriptional interference in avian retroviruses - implications for the promoter insertion model of leukaemogenesis. Nature 307: $241-245$.

Culotta, V. 1987. Ph.D. thesis, The transcriptionally active state of eukaryotic ribosomal RNA genes. Baltimore, Maryland. Johns Hopkins School of Medicine.

DeWinter, R.F. and T. Moss. 1987. A complex array of sequences enhances ribosomal transcription in $X$. laevis. $J$. Mol. Biol. 196: 813-827.

Grummt, I., U. Maier, A. Ohrlein, N. Hassouna, and J-P. Bachellerie. 1985. Transcription of mouse rDNA terminates downstream of the $3^{\prime}$ end of 28S RNA and involves interaction of factors with repeated sequences in the $3^{\prime}$ spacer. Cell 43: $801-810$.

Grummt, I., A. Kuhn, I. Bartsch, and H. Rosenbauer. 1986. A transcription terminator located upstream of the mouse rDNA initiation site affects rRNA synthesis. Cell 47: 901911.

Hackett, P.B., and W. Sauerbier. 1975. The transcriptional organization of the ribosomal RNA genes in mouse $\mathrm{L}$ cells. $J$. Mol. Biol. 91: 235-256.

Hausler, B. and R.L. Somerville. 1979. Interaction in vivo between strong closely spacer constitutive promoter. $J$. Mol. Biol. 127: 353-356.

Harrington, C.A. and D.M. Chikaraishi. 1987. Transcription of spacer sequences flanking the rat $45 \mathrm{~S}$ ribosomal DNA gene. Mol. Cell. Biol. 7: 314-325.

Henderson, S. and B. Sollner-Webb. 1986. A transcriptional terminator is a novel element of the promoter of the mouse 
ribosomal RNA gene. Cell 47: 891-900.

Johnson, P.J., J.M. Kooter, and P. Borst. 1987. Inactivation of transcription by UV irradiation of $T$. brucei provides evidence for a multicistronic transcription unit including a VSG gene. Cell 51: 273-281.

Kadesch, T. and P. Berg. 1986. Effects of the position of the simian virus 40 enhancer on expression of multiple transcription units in a single plasmid. Mol. Cell. Biol. 6: 25932601.

Kuhn, A. and I. Grummt. 1987. A novel promoter in the mouse rDNA spacer is active in vivo and in vitro. $E M B O \quad J$. 6: $3487-3492$.

Kurl, R. and S. Jacob. 1985. Characterization of a factor that can prevent random transcription of cloned DNA and its probable relationship to poly (ADP-ribose) polymerase. Nucleic Acids Res. 13: 89-101.

Lopata, M.A., D.W. Cleveland, and B. Sollner-Webb. 1984. High level transient expression of a chloramphenicol acetyl transferase gene by DEAE-dextran mediated DNA transfection coupled with a dimethyl sulfoxide or glycerol shock treatment. Nucleic Acids Res. 12: 5707-5717.

McStay, B. and R.H. Reeder. 1986. A terminator site for Xenopus RNA polymerase I also acts as an element of an adjacent promoter. Cell 47: 913-920.

Miller, K.G. and B. Sollner-Webb. 1981. Transcription of mouse rRNA genes by RNA polymerase I: in vitro and in vivo initiation and processing sites. Cell 27: 165-174.

Miller, K.G., J. Tower, and B. Sollner-Webb. 1985. A complex control region of the mouse rRNA gene directs accurate initiation by RNA polymerase I. Mol. Cell. Biol. 5: 554-562.

Mitchelson, K. and T. Moss. 1987. The enhancement of ribosomal transcription by the recycling of RNA polymerase I. Nucleic Acids Res. 15: 9577-9596.

Morgan, G.T., R.H. Reeder, and A.H. Bakken. 1983. Transcription in cloned spacers of Xenopus laevis ribosomal DNA. Proc. Natl. Acad. Sci. 80: 6490-6494.

Moss, T. 1983. A transcriptional function for the repetitive ribosomal spacer in Xenopus laevis. Nature 302: 223-228.

Proudfoot, N.J. 1986. Transcriptional interference and termination between duplicated $\alpha$-globin gene constructs suggests a novel mechanism for gene regulation. Nature 322: 562565.

Tower, J. and B. Sollner-Webb. 1987. Transcription of mouse rDNA is regulated by an activated form of RNA polymerase I. Cell 50: $873-883$.

Tower, J., V.C. Culotta, and B. Sollner-Webb. 1986. Factors and nucleotide sequences that direct ribosomal DNA transcription and their relationship to the stable transcription complex. Mol. Cell Biol. 6: 3451-3462.

Tower, J., S.L. Henderson, K. Dougherty, P.J. Wejksnora, and B. Sollner-Webb. 1989. An RNA polymerase I promoter located in the CHO and mouse rDNA spacers: functional analysis, factor, and sequence requirements. Mol. Cell. Biol. (in press).

Wandelt, C. and I. Grummt. 1983. Formation of stable preinitiation complexes is a prerequisite for ribosomal DNA transcription in vitro. Nucleic Acids Res. 11: 3795-3809. 


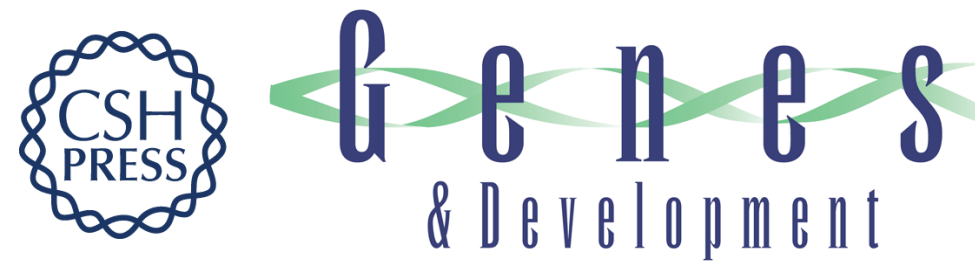

\section{The promoter-proximal rDNA terminator augments initiation by preventing disruption of the stable transcription complex caused by polymerase read-in.}

S L Henderson, K Ryan and B Sollner-Webb

Genes Dev. 1989, 3:

Access the most recent version at doi:10.1101/gad.3.2.212

References This article cites 31 articles, 7 of which can be accessed free at: http://genesdev.cshlp.org/content/3/2/212.full.html\#ref-list-1

License

Email Alerting Receive free email alerts when new articles cite this article - sign up in the box at the top Service right corner of the article or click here.

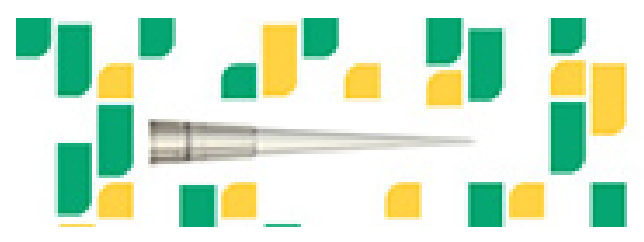

Focused on your science. 